\title{
THE APPLICATION OF BECK'S METHOD COMBINED WITH FEM AND TREFFTZ FUNCTIONS TO DETERMINE THE HEAT TRANSFER COEFFICIENT IN A MINICHANNEL
}

\author{
Beata Maciejewska \\ Kielce University of Technology, Department of Management and Computer Modelling, Kielce, Poland \\ e-mail: beatam@tu.kielce.pl
}

The aim of this study is to determine the heat transfer coefficient between the heated surface and the boiling fluid flowing in a minichannel on the basis of experimental data. The calculation model is based on Beck's method coupled with the FEM and Trefftz functions. The Trefftz functions used in the Hermite interpolation are employed to construct the shape functions in the FEM. The unknown local values of the heat transfer coefficient at the foil-fluid contact surface are calculated from Newton's law. The temperature of the heated foil and the heat flux on the foil surface are determined by solving a two-dimensional inverse heat conduction problem. The study is focused on the identification of the heat transfer coefficients in the subcooled boiling region and the saturated nucleate boiling region. The results are compared with the data obtained through the one-dimensional method. The investigations also reveal how the smoothing of measurement data affects calculation results.

Keywords: Beck's method, FEM, Trefftz functions, heat transfer coefficient, inverse heat conduction problem, flow boiling

\section{Introduction}

The main goal of this study is to determine the heat transfer coefficient at the interface between the heated minichannel wall and the boiling fluid flowing trought the minichannel. To identify this coefficient we need to know the wall temperature, the temperature gradient and the fluid temperature. The two-dimensional calculation model proposed for determining these quantities requires solving an inverse heat conduction problem. Inverse problems are problems in which the causes of a process are estimated by measuring the process results (Beck et al., 1985). Solutions to inverse problems are generally badly conditioned, which means that small changes in the input lead to large changes in the output (Tikhonov and Arsenin, 1977). Because of this property, inverse problems are much more difficult to solve than direct problems.

One of the classical methods used to solve inverse problems is the sensitivity coefficient method, also known as Beck's method or the sequential function specification method (Beck et al., 1985). This approach involves introducing sensitivity coefficients as a derivative of the measured quantity with respect to the identified quantity and transforming an inverse problem into several direct problems. The direct problems can then be solved using the finite difference method (Beck, 1970; Lin et al., 2008; Shi and Wang, 2009), the boundary element method (Kurpisz an Nowak, 1992; Le Niliot and Lefevre, 2004), the finite element method (Duda and Taler, 2009; Tseng et al., 1996), or the Trefftz method (Kruk and Sokała, 1999, 2000; Piasecka and Maciejewska, 2012). Although the sensitivity coefficient method is generally used to solve unsteady state problems, it can also be adapted to solve steady state problems (Kruk and Sokała, 1999; Piasecka and Maciejewska, 2012; Tseng et al., 1995).

The approach proposed by Trefftz (1926) seems particularly useful to deal with inverse problems. It involves approximating the unknown solution of a differential equation by means 
of a linear combination of functions strictly satisfying the differential equation. Such functions are known as Trefftz functions. Then, it is necessary to adjust the approximation function to match the boundary conditions and, additionally, the initial conditions in the case of unsteady state problems. Details of the method based on Trefftz functions can be found in (Ciałkowski and Grysa, 2009; Grysa and Maciejewska, 2013; Herrera, 2000; Hożejewska et al., 2009; Kompis et al., 2001; Li et al., 2006; Maciąg, 2011).

The application of Trefftz functions to construct shape functions in the finite element method and the use of this method to identify the boundary conditions in a steady-state problem are discussed in (Ciałkowski and Frąckowiak, 2002; Grysa et al., 2012; Piasecka and Maciejewska, 2013). Inverse unsteady state problems solved by means of the FEM with space-time basis functions are shown in (Ciałkowski, 2002; Maciejewska, 2004).

In this study, Beck's method combined with the FEM and Trefftz-type basis functions is used to solve the inverse heat conduction problem. This method allows us to directly determine the heat flux on the boundary surface; there is no need to differentiate the temperature function. In numerical calculations, differentiation of a function can lead to errors in results. The use of Beck's method connected with the Trefftz method, as proposed by Piasecka and Maciejewska (2012), was reported to be ineffective in a more complicated distribution of heat flux density on the boundary. It is assumed that the calculations should be performed by means of the FEM. The use of the Trefftz functions and the Hermite interpolation to construct the basis functions give satisfactory results. Details of this approach will be described in the next Sections. The Hermite interpolation was shown by Kincaid and Cheney (2002).

\section{Experimental research}

The calculation of the heat transfer coefficient has been performed using the experimental data obtained from the experimental setup described in detail in (Piasecka, 2014a,c, 2015; Piasecka and Maciejewska, 2015).

The main element of the experimental setup was a cuboidal minichannel $1 \mathrm{~mm} \times 40 \mathrm{~mm} \times 360 \mathrm{~mm}$. FC-72 was used as the working fluid flowing up the minichannel with the velocity $u=0.17 \mathrm{~m} / \mathrm{s}$. The average mass flux $G$ was $282 \mathrm{~kg} /\left(\mathrm{m}^{2} \mathrm{~s}\right)$, Reynolds number Re was 950, inlet liquid subcooling defined as the difference between the saturation temperature at the minichannel inlet and the fluid temperature at the minichannel inlet $\Delta T_{s u b, i n}$ was $36 \mathrm{~K}$. One of the minichannel walls was a heated foil made of Haynes 230 alloy. Because of the electrical properties of the material, it was possible to produce a large heat flux at a relatively small surface area of the foil. On the side in contact with the fluid, the heated foil had evenly distributed microcavities produced by laser machining (Piasecka, 2014b; Piasecka and Maciejewska, 2015).

The heated foil was separated from the surroundings with a glass panel. The surface of the foil in contact with the glass was covered with a thin layer of thermochromic liquid crystals.

During the experiments, the quantities were measured in the steady state. The measurement data included:

- heat flow parameters:

- local temperature of the heated foil at the surface in contact with the glass panel determined from the distribution of hues on the liquid crystal layer using the method described by Piasecka (2013);

- fluid temperature at the minichannel inlet $T_{f, i n}$ and the fluid temperature at the minichannel outlet $T_{f, \text { out }}$, measured with K-type thermocouples linked to the data acquisition station;

- volumetric flow rate $Q_{V}$ measured with rotameters; 
- pressure at the minichannel inlet $p_{\text {in }}$ and pressure at the minichannel outlet $p_{\text {out }}$ measured with pressure transducers linked to the data acquisition station;

- electrical parameters:

- drop in voltage $\Delta U$ along the length of the heated foil, measured with a voltmeter;

- electric current supplied to the heated foil $I$ measured with an ammeter;

- flow structures.

The capacity of the heat source (volumetric heat flux) has been determined from the formula

$$
q_{V}=\frac{I \Delta U}{A_{F} \delta_{F}}=\frac{q_{w}}{\delta_{F}}
$$

where $I$ is the current supplied to the heated foil, $\Delta U$ - drop in voltage along the length of the heated foil, $A_{F}$ - surface area of the heated foil in contact with the fluid, $\delta_{F}$ - thickness of the heated foil, $q_{w}$ - heat flux.

The numerical calculations have been performed using the measurement data presented in Fig. 1 and Table 1. The other quantities used in the analysis are: surface area of the heated foil in contact with the fluid $A_{F}=0.0234 \mathrm{~m}^{2}$, thickness of the heated foil $\delta_{F}=0.00016 \mathrm{~m}$, thickness of the glass panel $\delta_{G}=0.006 \mathrm{~m}$, length of the glass panel $L=0.35 \mathrm{~m}$, thermal conductivity coefficient of the foil $\lambda_{F}=8.3 \mathrm{~W} /(\mathrm{mK})$ and thermal conductivity coefficient of the glass panel $\lambda_{G}=0.71 \mathrm{~W} /(\mathrm{mK})$.

(a)

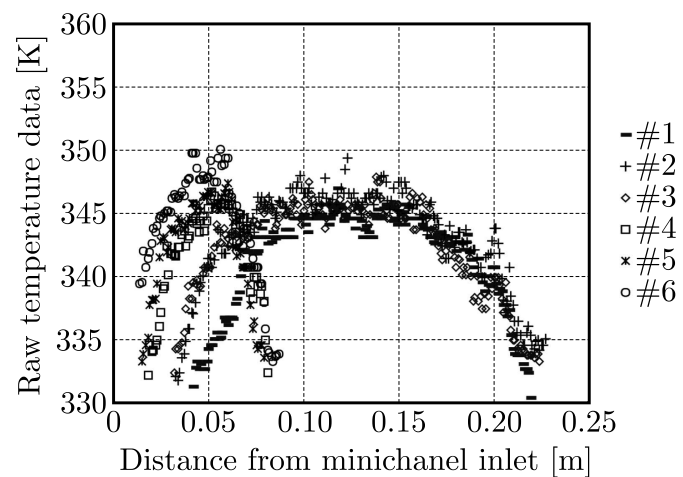

(b)

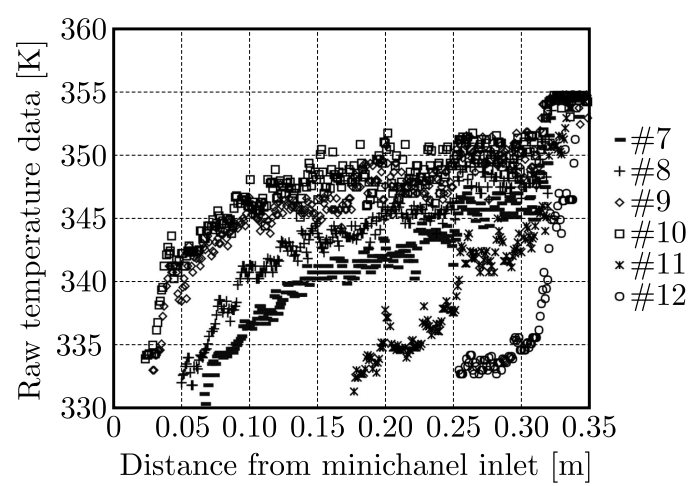

Fig. 1. Raw temperature data obtained from measurements at the foil-glass interface, corresponding to: (a) subcooled boiling region, (b) saturated nucleate boiling region

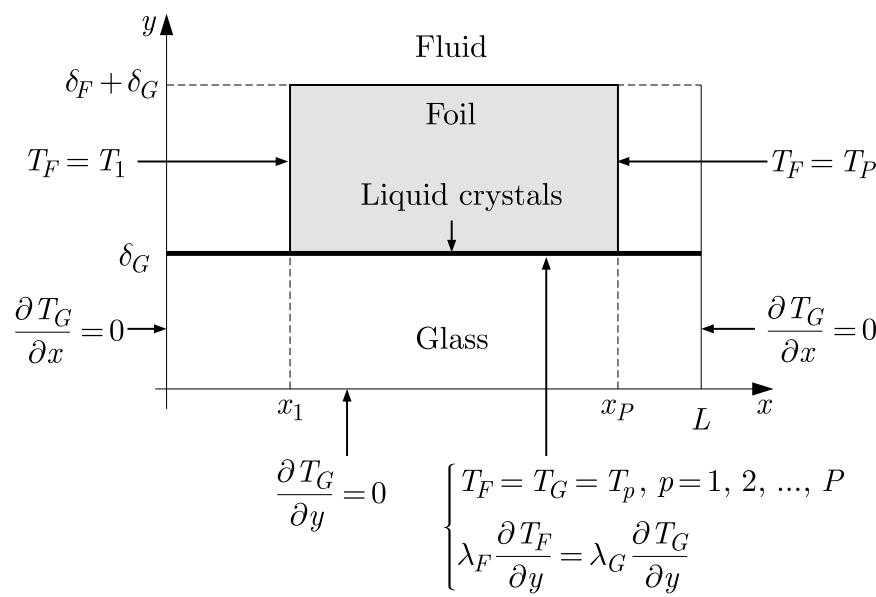

Fig. 2. The boundary conditions (note: figure not to scale) 
Table 1. Measurement data used in the calculations: $I$ - current supplied to the heated foil, $\Delta U$ - drop in voltage along the length of the heated foil, $T_{f}$ - fluid temperature, $p$ - pressure; indexes in, out refer to minichannel inlet and outlet

\begin{tabular}{|c|c|c|c|c|c|c|}
\hline $\begin{array}{c}\text { Setting } \\
\text { number }\end{array}$ & $\begin{array}{c}I \\
{[\mathrm{~A}]}\end{array}$ & $\begin{array}{c}\Delta U \\
{[\mathrm{~V}]}\end{array}$ & $\begin{array}{c}T_{f, \text { in }} \\
{[\mathrm{K}]}\end{array}$ & $\begin{array}{c}T_{f, \text { out }} \\
{[\mathrm{K}]}\end{array}$ & $\begin{array}{c}p_{\text {in }} \\
{[\mathrm{Pa}]}\end{array}$ & $\begin{array}{c}p_{\text {out }} \\
{[\mathrm{Pa}]}\end{array}$ \\
\hline \hline$\# 1$ & 39.8 & 5.93 & 301.15 & 310.65 & 119850 & 110950 \\
\hline$\# 2$ & 41 & 6.03 & 300.95 & 311.85 & 119150 & 113450 \\
\hline$\# 3$ & 42.6 & 6.14 & 300.85 & 312.65 & 123250 & 114550 \\
\hline$\# 4$ & 44 & 6.84 & 300.85 & 314.35 & 124150 & 113950 \\
\hline$\# 5$ & 45.2 & 6.47 & 300.55 & 314.85 & 123650 & 114750 \\
\hline$\# 6$ & 46.60 & 6.54 & 300.55 & 315.65 & 123950 & 117450 \\
\hline$\# 7$ & 63.20 & 8.33 & 299.95 & 334.35 & 132050 & 124550 \\
\hline$\# 8$ & 64.40 & 8.53 & 300.25 & 335.95 & 140550 & 119950 \\
\hline$\# 9$ & 65.40 & 8.60 & 300.35 & 337.85 & 139650 & 132350 \\
\hline$\# 10$ & 61.60 & 8.19 & 301.25 & 338.05 & 140750 & 133150 \\
\hline$\# 11$ & 51.60 & 7.05 & 301.75 & 330.75 & 127950 & 119750 \\
\hline$\# 12$ & 48.20 & 6.79 & 300.75 & 326.05 & 125650 & 117050 \\
\hline
\end{tabular}

The numerical calculations have been performed also for the smoothed temperature data (see Fig. 2). The data was smoothed by means of the approximating polynomial based on the Trefftz functions using the least squares method (Grysa et al., 2012).

\section{Mathematical model}

Two-dimensional stationary heat transfer in the minichannel described in Cartesian coordinates $x, y$ is assumed in the investigations. The $x$ coordinate refers to the fluid flow direction and the $y$ coordinate relates to thickness of the heated foil and the glas panel. In this investigation, variation in temperature along width of the minichannel is neglected.

The local values of the heat transfer coefficient between the heated foil and the boiling fluid flowing in the minichannel are calculated using Newton's law.

$$
\alpha_{2 D}(x)=\frac{q(x)}{T_{F}\left(x, \delta_{G}+\delta_{F}\right)-T_{f}(x)}
$$

where $q$ is the heat flux transferred from the heated foil to the fluid, $T_{F}$ - temperature of the foil, with $q$ and $T_{F}$ determined by solving the inverse heat conduction problem in the heated foil, $\delta_{G}$ - thickness of the glass panel, $\delta_{F}$ - thickness of the foil, $T_{f}$ - temperature of the fluid, with $T_{f}(x)=T_{l}(x)$ in the subcooled boiling region and $T_{f}(x)=T_{\text {sat }}(x)$ in the saturated nucleate boiling region, $T_{l}$ - liquid temperature calculated on the assumption of a linear distribution of liquid temperature along the minichannel from the temperature $T_{f, \text { in }}$ to the temperature $T_{f, \text { out }}$, and $T_{\text {sat }}$-saturation temperature determined on the assumption of a linear distribution of fluid pressure along the minichannel (Piasecka and Maciejewska, 2015; Piasecka et al., 2017).

The mathematical model is based on the model presented by Hożejowska and Piasecka (2014). For the purpose of the FEM, changes in the determinancy domain of the differential equation and in the boundary conditions are taken into account.

The temperature of the heated foil satisfies the Poisson equation

$$
\begin{aligned}
& \frac{\partial^{2} T_{F}}{\partial x^{2}}+\frac{\partial^{2} T_{F}}{\partial y^{2}}=-\frac{q_{V}}{\lambda_{F}} \\
& \text { for } \quad(x, y) \in \Omega_{F}=\left\{(x, y) \in R^{2}: x_{1}<x<x_{P}, \delta_{G}<y<\delta_{G}+\delta_{F}\right\}
\end{aligned}
$$


and the boundary conditions (see Fig. 2)

$$
\begin{array}{ll}
T_{F}\left(x, \delta_{G}\right)=T_{G}\left(x, \delta_{G}\right) & \lambda_{F} \frac{\partial T_{F}}{\partial y}\left(x, \delta_{G}\right)=\lambda_{G} \frac{\partial T_{G}}{\partial y}\left(x, \delta_{G}\right) \\
T_{F}\left(x_{1}, y\right)=T_{1} & T_{F}\left(x_{P}, y\right)=T_{P}
\end{array}
$$

and

$$
T_{F}\left(x_{p}, \delta_{G}\right)=T_{p} \quad \text { for } \quad p=1,2, \ldots, P
$$

where $x_{1}$ is the location of the first measurement point at the boundary $y=\delta_{G}, x_{P}$ - location of the last measurement point, $P$ - number of measurements, $T_{p}$ - measured temperature, $\lambda_{F}$ and $\lambda_{G}$-thermal conductivity coefficients of the foil and glass, respectively, $q_{V}, \delta_{G}, \delta_{F}$ have the same denotations as in Eqs. (2.1) and (3.1).

The temperature of the glass panel, as in (Hożejowska and Piasecka, 2014), has been determined by solving the direct heat conduction problem

$$
\frac{\partial^{2} T_{G}}{\partial x^{2}}+\frac{\partial^{2} T_{G}}{\partial y^{2}}=0 \quad \text { for } \quad(x, y) \in \Omega_{G}=\left\{(x, y) \in R^{2}: 0<x<L, 0<y<\delta_{G}\right\}
$$

and

$$
\begin{array}{llrl}
\frac{\partial T_{G}}{\partial y}(x, 0) & =0 & \frac{\partial T_{G}}{\partial x}(0, y)=0 & \frac{\partial T_{G}}{\partial x}(L, y)=0 \\
T_{G}\left(x_{p}, \delta_{G}\right)=T_{p} & \text { for } \quad p=1,2, \ldots, P &
\end{array}
$$

where $L$ denotes length of the glass panel, $\delta_{G}, x_{P}, P$ have the same denotations as in Eqs. (3.2)-(3.4).

The inverse problem, Eqs. (3.2)-(3.4), has been solved using Beck's method combined with the FEM and Trefftz functions. With the Trefftz functions used, the approximate functions exactly satisfy the governing differential equations. The direct problem, Eqs. (3.5) and (3.6), has been solved by means of the Trefftz method described by Hożejowska et al. (2015).

\section{Beck's method coupled with the FEMT}

Beck's method (Beck et al., 1985) involves converting an inverse problem into several direct problems by applying the so-called sensitivity coefficients. Since the heat flux at the boundary is the unknown quantity here, it is essential to determine the sensitivity coefficients as derivatives of temperature with respect to the unknown flux.

The calculations have been performed assuming the heat flux $q$ at the boundary $y=\delta_{G}+\delta_{F}$ for $x_{1} \leqslant x \leqslant x_{P}$ in the form

$$
q=\sum_{m=1}^{L 1}\left[U\left(x-x_{m}\right)-U\left(x-x_{m+1}\right)\right] q_{m}
$$

where $U$ is the unit step function (the Heaviside function), while $q_{m}$ for $m=1,2, \ldots, L 1$ take constant values (Kruk and Sokała, 1999). The same partition of the boundary $y=\delta_{G}+\delta_{F}$, $x \in\left\langle x_{1}, x_{P}\right\rangle$ into $L 1$ parts will also be used in the FEM.

The temperature $T_{F}$ dependent on the $q_{m}$ fluxes for $m=1,2, \ldots, L 1$ at the boundary $y=\delta_{G}+\delta_{F}$ for $x_{1} \leqslant x \leqslant x_{P}$, like in (Kruk and Sokała, 1999), is expanded into a Taylor series 
about a fixed point $\left(q_{01}, \ldots, q_{0 L 1}\right)$. Since higher order derivatives disappear in linear problems, we obtain the formula

$$
T_{F}\left(x, y, q_{1}, \ldots, q_{L 1}\right)=T_{F}\left(x, y, q_{01}, \ldots, q_{0 L 1}\right)+\left.\sum_{m=1}^{L 1} \frac{\partial T_{F}}{\partial q_{m}}\right|_{q_{m}=q_{0 m}}\left(q_{m}-q_{0 m}\right)
$$

After introducing the denotations $\Theta_{F}(x, y)=T_{F}\left(x, y, q_{01}, \ldots, q_{0 L 1}\right)$ and $Z_{m}(x, y)=$ $\left.\left(\partial T_{F} / \partial q_{m}\right)\right|_{q_{m}=q_{0 m}}$, expression (4.2) is written as

$$
T_{F}\left(x, y, q_{1}, \ldots, q_{L 1}\right)=\Theta_{F}(x, y)+\sum_{m=1}^{L 1} Z_{m}(x, y)\left(q_{m}-q_{0 m}\right)
$$

where $Z_{m}(x, y)$, for $m=1,2, \ldots, L 1$ are the sensitivity coefficients.

$\Theta_{F}(x, y)$ and $Z_{m}(x, y)$ for $m=1,2, \ldots, L 1$ in the domain $\Omega_{F}$ are determined by solving $1+L 1$ direct problems that arise after substituting Eq. (4.3) into Eq. (3.2) and boundary conditions Eq. (3.3)

$$
\begin{array}{lcc}
\frac{\partial^{2} \Theta_{F}}{\partial x^{2}}+\frac{\partial^{2} \Theta_{F}}{\partial y^{2}}=-\frac{q_{V}}{\lambda_{F}} & \text { for } & (x, y) \in \Omega_{F} \\
\Theta_{F}\left(x, \delta_{G}\right)=T_{G}\left(x, \delta_{G}\right) & \lambda_{F} \frac{\partial \Theta_{F}}{\partial y}\left(x, \delta_{G}\right)=\lambda_{G} \frac{\partial T_{G}}{\partial y}\left(x, \delta_{G}\right) \\
\frac{\partial \Theta_{F}}{\partial y}\left(x, \delta_{G}+\delta_{F}\right)=0 & \Theta_{F}\left(x_{1}, y\right)=T_{1} & \Theta_{F}\left(x_{P}, y\right)=T_{P}
\end{array}
$$

and

$$
\begin{array}{lc}
\frac{\partial^{2} Z_{m}}{\partial x^{2}}+\frac{\partial^{2} Z_{m}}{\partial y^{2}}=0 & \text { for } m=1,2, \ldots L 1 \quad \text { and } \quad(x, y) \in \Omega_{F} \\
Z_{m}\left(x_{1}, y\right)=0 & Z_{m}\left(x_{P}, y\right)=0 \\
\frac{\partial Z_{m}}{\partial y}\left(x, \delta_{G}\right)=0 & -\lambda_{F} \frac{\partial Z_{m}}{\partial y}\left(x, \delta_{G}+\delta_{F}\right)=U\left(x-x_{m}\right)-U\left(x-x_{m+1}\right)
\end{array}
$$

Condition (3.4) will be used in the subsequent calculations.

The functions $\Theta_{F}$ and $Z_{m}$ for $m=1,2, \ldots, L 1$ have been determined using the finite element method combined with the Trefftz-type basis functions (FEMT), as described in (Piasecka and Maciejewska, 2013). In this paper, the partition of the domain $\Omega_{F}$ into finite elements is closely linked to the partition of the boundary $y=\delta_{G}+\delta_{F}, x \in\left\langle x_{1}, x_{P}\right\rangle$ into $L 1$ parts, like in Eq. (4.1). The basis functions $f_{j k}(x, y), g_{j k}(x, y), h_{j k}(x, y)$ constructed with the Hermite interpolation (Kincaid and Cheney, 2002), have the following properties in nodes $\left(x_{i}, y_{i}\right)$

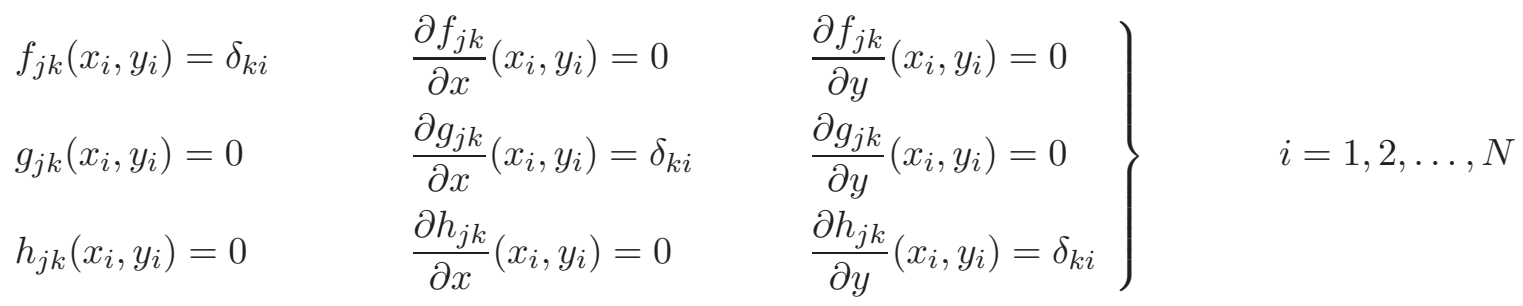

where $j$ is the element number, $k$ - number of the basis function in the $j$-th element, $N$-number of nodes in the $j$-th element, $\delta_{k i}$ - Kronecker delta.

Three nodal parameters are associated with each interpolation node: the value of the function at a node, the value of the partial derivative with respect to $x$, and the value of the partial derivative with respect to $y$. 
In each element $\Omega_{F}^{j}$, the function $\Theta_{F}(x, y)$ is approximated by means of a linear combination of the basis functions

$$
\begin{aligned}
& \Theta_{F}^{j}(x, y)=u(x, y)+\sum_{k=1}^{N}\left\{\left[a_{n}-u\left(x_{n}, y_{n}\right)\right] f_{j k}(x, y)+\left[b_{n}-u_{x}^{\prime}\left(x_{n}, y_{n}\right)\right] g_{j k}(x, y)\right. \\
& \left.\quad+\left[c_{n}-u_{y}^{\prime}\left(x_{n}, y_{n}\right)\right] h_{j k}(x, y)\right\}
\end{aligned}
$$

where $u(x, y)$ is the particular solution of equation $(4.4)_{1}, n$-number of the node in the whole domain $\Omega_{F}, a_{n}$-value of the unknown function at the $n$-th node of the domain $\Omega_{F}, b_{n}$-value of the partial derivative of the unknown function with respect to $x$ at the $n$-th node of the domain $\Omega_{F}, c_{n}$ - value of the partial derivative of the unknown function with respect to $y$ at the $n$-th node of the domain $\Omega_{F}, f_{j k}(x, y), g_{j k}(x, y)$ and $h_{j k}(x, y)$ - basis functions, $j, k, N$ have the same denotations as in Eqs. (4.6).

The unknown coefficients $a_{n}, b_{n}, c_{n}$ in linear combination (4.7) have been calculated, like in (Piasecka and Maciejewska, 2013), by minimizing the functional $J$ which describes the mean square error of fit of the approximate function to the boundary conditions and the difference between the values of the approximate function at the common edges of the adjacent elements, and in this calculations has the form

$$
\begin{aligned}
J= & \sum_{j=1}^{L 1} \int_{x_{j}}^{x_{j+1}}\left[\Theta_{F}^{j}\left(x, \delta_{G}\right)-T_{G}^{j}\left(x, \delta_{G}\right)\right]^{2} d x+\sum_{j=1}^{L 1} \int_{x_{j}}^{x_{j+1}}\left[\lambda_{F} \frac{\partial \Theta_{F}^{j}}{\partial y}\left(x, \delta_{G}\right)-\lambda_{G} \frac{\partial T_{G}^{j}}{\partial y}\left(x, \delta_{G}\right)\right]^{2} d x \\
& +\sum_{j=1}^{L 1} \int_{x_{j}}^{x_{j+1}}\left[\frac{\partial \Theta_{F}^{j}}{\partial y}\left(x, \delta_{G}+\delta_{F}\right)\right]^{2} d x+\sum_{i=0}^{L 2-1} \int_{\delta_{G}+y_{i+1}}^{\delta_{G}+y_{i+2}}\left[\Theta_{F}^{1+i L 1}\left(x_{1}, y\right)-T_{1}\right]^{2} d y \\
& +\sum_{i=0}^{L 2-1} \int_{\delta_{G}+y_{i+1}}^{\delta_{G}+y_{i+2}}\left[\Theta_{F}^{(i+1) L 1}\left(x_{P}, y\right)-T_{P}\right]^{2} d y \\
& +\sum_{i=0}^{L 2-1} \sum_{j=1}^{L 1-1} \int_{\delta_{G}+y_{i+1}}^{\delta_{G}+y_{i+2}}\left[\Theta_{F}^{j+i L 1}\left(x_{j+1}, y\right)-\Theta_{F}^{j+1+i L 1}\left(x_{j+1}, y\right)\right]^{2} d y \\
& +\sum_{i=0}^{L 2-1} \sum_{j=1}^{L 1-1} \int_{\delta_{G}+y_{i+1}}^{\delta_{G}+y_{i+2}}\left[\frac{\partial \Theta_{F}^{j+i L 1}}{\partial x}\left(x_{j+1}, y\right)-\frac{\partial \Theta_{F}^{j+1+i L 1}}{\partial x}\left(x_{j+1}, y\right)\right]^{2} d y \\
& +\sum_{i=1}^{L 2-1} \sum_{j=1}^{L 1} \int_{x_{j}}^{x_{j+1}}\left[\Theta_{F}^{j+(i-1) L 1}\left(x, \delta_{G}+y_{i+1}\right)-\Theta_{F}^{j+i L 1}\left(x, \delta_{G}+y_{i+1}\right)\right]^{2} d x \\
& +\sum_{i=0}^{L 2-1} \sum_{j=1}^{L 1} \int_{x_{j}}^{x_{j+1}}\left[\frac{\partial \Theta_{F}^{j+(i-1) L 1}}{\partial y}\left(x, \delta_{G}+y_{i+1}\right)-\frac{\partial \Theta_{F}^{j+i L 1}}{\partial y}\left(x, \delta_{G}+y_{i+1}\right)\right]^{2} d x
\end{aligned}
$$

Similarly, the solutions to the $L 1$ direct problems give the sensitivity coefficients $Z_{m}$ for $m=1,2, \ldots, L 1$.

The values of $q_{m}$ for $m=1,2, \ldots, L 1$ in expression (4.3) have been calculated by minimizing the functional $J_{F}^{P}$ that describes the mean square error between the values of the function $T_{F}\left(x, y, q_{1}, \ldots, q_{L 1}\right)$ at the measurement points and temperature measurements

$$
J_{F}^{P}=\sum_{p=1}^{P}\left[T_{F}\left(x_{p}, y_{p}, q_{1}, \ldots, q_{L 1}\right)-T_{p}\right]^{2}
$$




\section{Calculation results}

The values of the heat transfer coefficient have been obtained by solving the inverse heat conduction problem through Beck's method coupled with the finite element method in which the Trefftz functions were used as basis functions. The values of this coefficient were determined in the subcooled boiling region and in the saturated nucleate boiling region.

The calculations were performed using the raw temperature data presented in Fig. 1 as well as the smoothed temperature data. In both approaches, two variants of the partition of the boundary $y=\delta_{G}+\delta_{F}$ for $x \in\left\langle x_{1}, x_{P}\right\rangle$ into subdomains were considered. In variant one, the boundary was partitioned into $L 1=10$ subdomains, while in variant two, it was partitioned into $L 1=20$ subdomains. In neither case the domain $\Omega_{F}$ was partitioned in the $y$-direction. The four Hermite interpolation nodes were placed at the vertices of rectangular elements of the mesh. As three nodal parameters were associated with each interpolation node, the basis functions were constructed using 12 Trefftz functions. The particular solution to Eq. (4.4) $)_{1}$ was written in the following form $u(x, y)=-0.25 q_{V} \lambda_{F}^{-1}\left(y^{2}+x^{2}\right)$. The calculations were performed using the data from 12 settings shown in Fig. 1 as well as Table 1 . The heat transfer coefficients as a function of distance from the minichannel inlet are shown in Figs. 3-5.
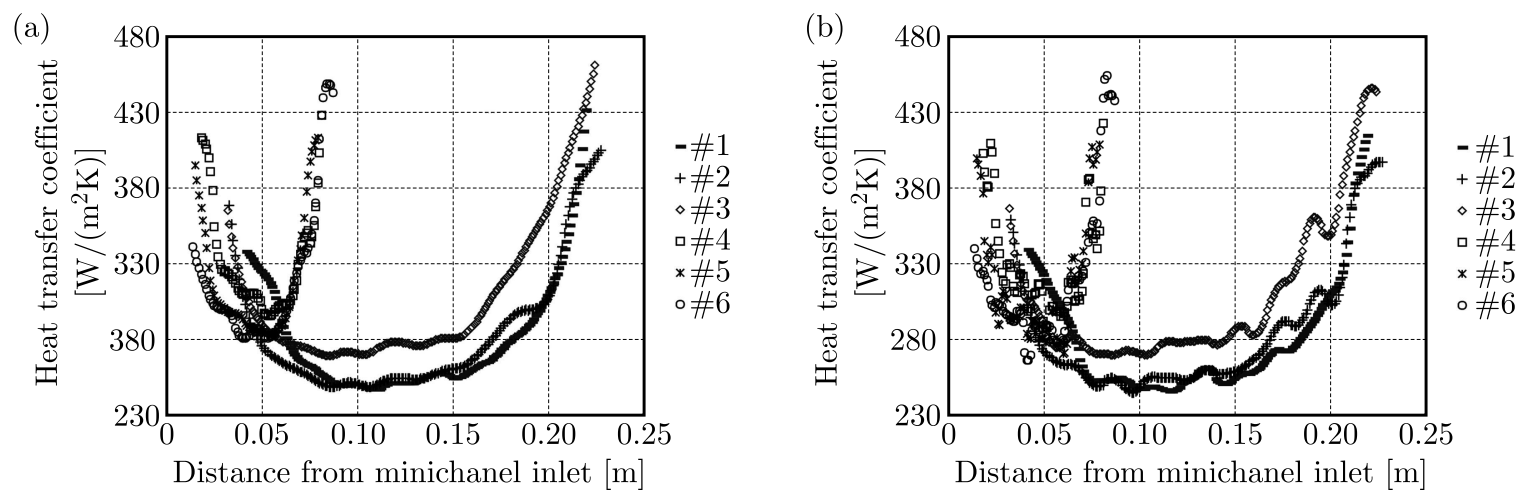

Fig. 3. Heat transfer coefficients in the subcooled boiling region vs. distance from the minichannel inlet obtained on the basis of the raw temperature data with the boundary partitioned into:

(a) $L 1=10$ subdomains, (b) $L 1=20$ subdomains
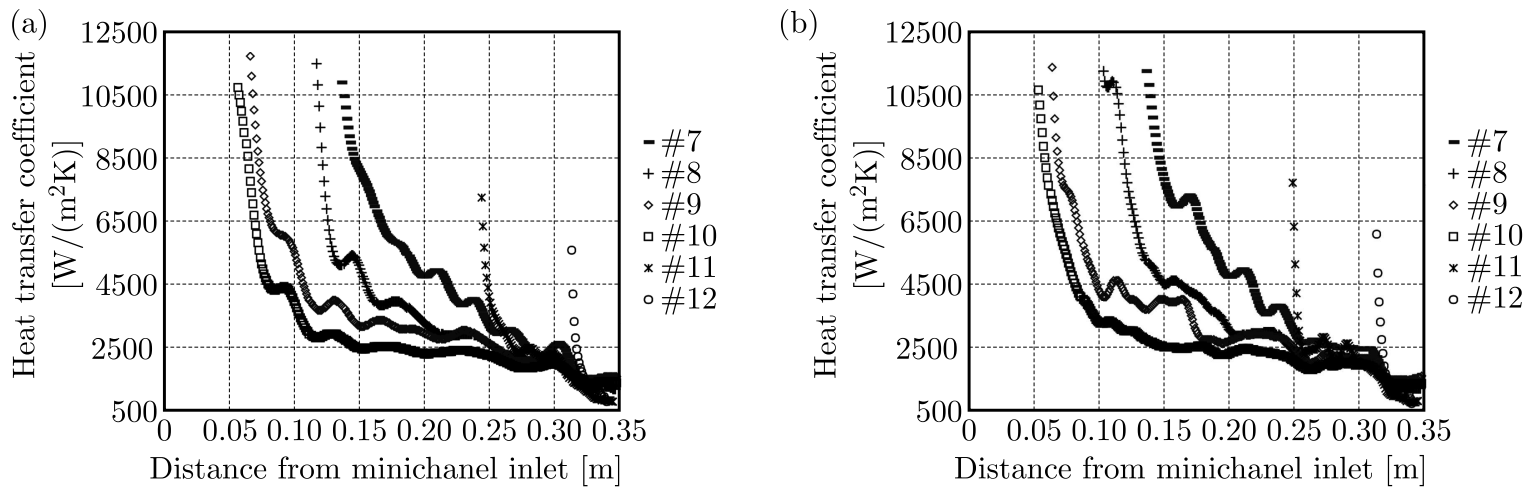

Fig. 4. Heat transfer coefficients in the saturated nucleate boiling region vs. distance from the minichannel inlet obtained on the basis of the raw temperature data with the boundary partitioned into: (a) $L 1=10$ subdomains, (b) $L 1=20$ subdomains

The relative differences between the values of the heat transfer coefficients obtained for both variants of the boundary partition into $L 1=10$ subdomains and $L 1=20$ subdomains were calculated according to formula (5.1) and shown in Table 2 

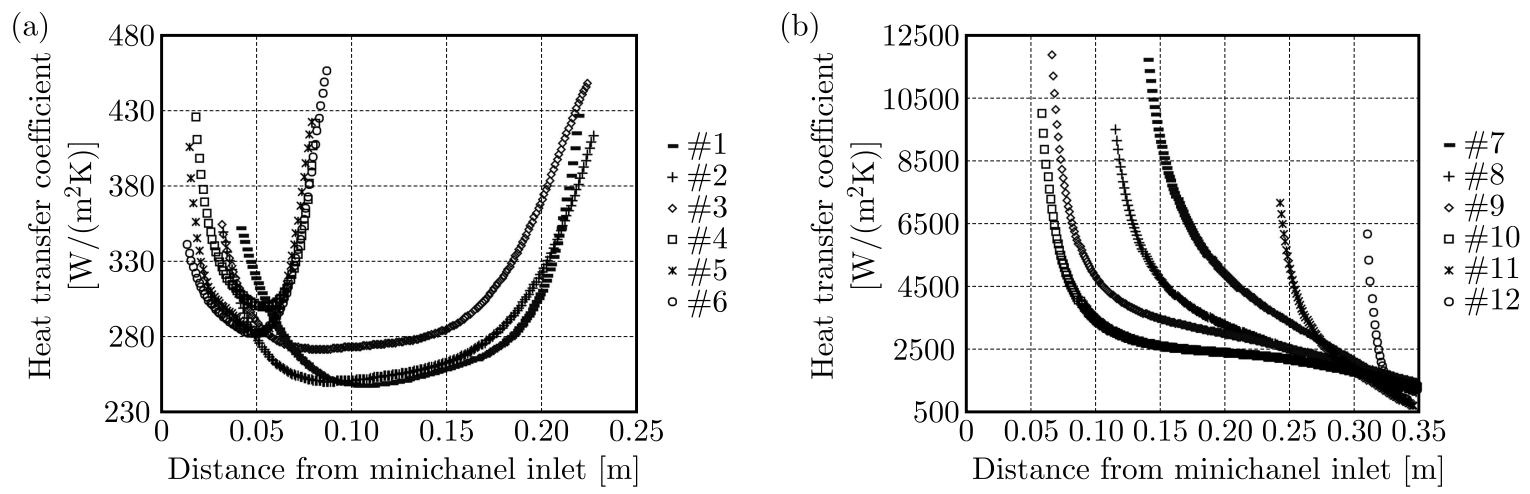

Fig. 5. Heat transfer coefficients vs. distance from the minichannel inlet obtained on the basis of the smoothed temperature data with the boundary partitioned into $L 1=10$ subdomains: (a) in the saturated nucleate boiling region, (b) in the subcooled boiling region

$$
\sigma_{i}=\frac{1}{P} \sum_{p=1}^{P} \sqrt{\frac{\left[\alpha_{2 D}^{i, L 1=10}\left(x_{p}\right)-\alpha_{2 D}^{i, L 1=20}\left(x_{p}\right)\right]^{2}}{\left\{\min \left[\alpha_{2 D}^{i, L 1=10}\left(x_{p}\right), \alpha_{2 D}^{i, L 1=20}\left(x_{p}\right)\right]\right\}^{2}}} \quad i=\text { Raw, Smoo }
$$

where $P$ denotes the number of measurements, $\alpha_{2 D}^{i, L 1=10}$ and $\alpha_{2 D}^{i, L 1=20}$ are values of the heat transfer coefficients calculated for $L 1=10$ and $L 1=20$ subdomains, respectively, indexes $i=R a w$ and $i=S m o o$ refer to the calculations based on the raw measurement data and the smoothed temperature data, respectively. From Table 2, it is evident that the greatest differences between the values of the heat transfer coefficients occurred at setting \#11 when the raw measurement data was used. Since there are very small differences between the values of the heat transfer coefficient obtained from the smoothed data for the case when the domain is divided into $L 1=10$ subdomains and those reported for the division into $L 1=20$ subdomains, Fig. 5 shows only the results obtained for $L 1=10$.

Table 2. Relative differences between the values of the heat transfer coefficient obtained for both variants of the boundary partition into $L 1=10$ subdomains and $L 1=20$ subdomains using the raw and smoothed temperature data

\begin{tabular}{|c|c|c|c|c|c|c|c|c|c|c|c|c|}
\hline & \multicolumn{6}{|c|}{ Subcooled boiling region } & \multicolumn{6}{|c|}{ Saturated nucleate boiling region } \\
\hline & \multicolumn{12}{|c|}{ Setting number } \\
\hline & $\# 1$ & $\# 2$ & $\# 3$ & $\# 4$ & $\# 5$ & $\# 6$ & $\# 7$ & $\# 8$ & $\# 9$ & $\# 10$ & $\# 11$ & $\# 12$ \\
\hline$\sigma_{\text {Raw }}[\%]$ & 1.33 & 1.23 & 1.2 & 3.11 & 2.98 & 2.07 & 5.54 & 5.73 & 8.55 & 5.1 & 12.57 & 9.32 \\
\hline$\sigma_{\text {Smoo }}[\%]$ & 0.08 & 0.12 & 0.08 & 0.04 & 0.05 & 0.4 & 1.9 & 1.57 & 2.8 & 2.76 & 0.17 & 0.12 \\
\hline
\end{tabular}

The obtained results are in agreement with the data presented in (Grysa et al., 2012; Hożejowska and Piasecka, 2014; Hożejowska et al., 2009; Ozer et al., 2011; Piasecka and Maciejewska, 2012, 2013, 2015; Piasecka et al., 2017), which are provided in Table 3. The values of the heat transfer coefficient are high in the saturated nucleate boiling region (like in Hożejowska and Piasecka, 2014; Piasecka and Maciejewska, 2015, Piasecka et al., 2016); they are much lower in the subcooled boiling region (like in Grysa et al., 2012; Hożejowska et al., 2009; Ozer et al., 2011; Piasecka and Maciejewska, 2012, 2013, 2015). The experimental parameters provided in Section 2 reported for the minichannel in the subcooled boiling region are most similar to the data described by Piasecka and Maciejewska (2012); while in the saturated nucleate boiling region resembled those discussed by Hożejowska and Piasecka (2014). The values of the heat transfer coefficient shown in Figs. 3-5 are not very different from those presented in (Piasecka and Maciejewska, 2012) and (Hożejowska and Piasecka, 2014). 
Table 3. Experimental data and heat transfer coefficients presented in (Grysa et al., 2012 [7]; Hożejowska and Piasecka, 2014 [11]; Hożejowska et al., 2009 [12]; Ozer et al., 2011 [23]; Piasecka and Maciejewska, 2012 [29], 2013 [30], 2015 [31]; Piasecka et al., 2017 [32])

\begin{tabular}{|c|c|c|c|c|c|c|c|c|c|}
\hline & \multicolumn{6}{|c|}{ Subcooled boiling region } & \multicolumn{3}{|c|}{$\begin{array}{l}\text { Saturated nucleate } \\
\text { boiling region }\end{array}$} \\
\hline Reference & $\overline{[\overline{[7]}}$ & $\overline{[12]}$ & $\overline{[23]}$ & $\overline{[29]}$ & $\overline{[30]}$ & $\overline{[31]}$ & $\overline{[11]}$ & $\overline{[31]}$ & $\overline{[32]}$ \\
\hline $\begin{array}{c}\text { No. of experim. } \\
\text { analysed }\end{array}$ & 1 & 1 & 2 & 3 & 1 & 3 & 1 & 3 & 2 \\
\hline Working fluid & R 123 & R 123 & $\begin{array}{c}\text { Novec } \\
649 \\
\end{array}$ & FC-72 & FC-72 & FC-72 & FC-72 & FC-72 & FC-72 \\
\hline $\begin{array}{l}\text { Minichannel } \\
\text { dimensions } \\
\text { dept, width, } \\
\text { length [mm] }\end{array}$ & $\begin{array}{c}1,40, \\
300\end{array}$ & $\begin{array}{c}1,40, \\
360\end{array}$ & $\begin{array}{l}1,2, \\
357\end{array}$ & $\begin{array}{c}1,60, \\
360\end{array}$ & $\begin{array}{c}1,40, \\
360\end{array}$ & $\begin{array}{c}1,40, \\
360\end{array}$ & $\begin{array}{c}1,40 \\
360\end{array}$ & $\begin{array}{c}1,40, \\
360\end{array}$ & $\begin{array}{l}1.7,24, \\
360\end{array}$ \\
\hline $\begin{array}{c}\text { Spatial } \\
\text { orientation }\end{array}$ & vert. & vert. & $\begin{array}{c}\text { hori- } \\
\text { zontal }\end{array}$ & $\begin{array}{l}\text { Exp. 1: } \\
\text { vert. } \\
\text { Exp. 2: } \\
\text { horiz. } \\
\text { Exp. 3: } \\
\text { horiz. }\end{array}$ & vert. & $\begin{array}{c}\text { Exp. 1: } \\
\text { vert. } \\
\text { Exp. 2: } \\
\text { horiz. } \\
\text { Exp. 3: } \\
\text { horiz. }\end{array}$ & vert. & $\begin{array}{c}\text { Exp. 1: } \\
\text { vert. } \\
\text { Exp. 2: } \\
\text { horiz. } \\
\text { Exp. 3: } \\
\text { horiz. }\end{array}$ & $\begin{array}{c}\text { Exp.1: } \\
\text { vert. } \\
\text { Exp. 2: } \\
\text { vert. }\end{array}$ \\
\hline $\begin{array}{c}\text { Type of heated } \\
\text { surface }\end{array}$ & smooth & smooth & smooth & smooth & $\begin{array}{c}\text { enhan- } \\
\text { ced }\end{array}$ & $\begin{array}{c}\text { enhan- } \\
\text { ced }\end{array}$ & $\begin{array}{c}\text { enhan- } \\
\text { ced }\end{array}$ & $\begin{array}{l}\text { enhan- } \\
\text { ced }\end{array}$ & $\begin{array}{c}\text { enhan- } \\
\text { ced }\end{array}$ \\
\hline $\begin{array}{c}\text { Heat flux } \\
q_{w}\left[\mathrm{~kW} / \mathrm{m}^{2}\right]\end{array}$ & $25.4-37.6$ & $\begin{array}{l}14.0, \\
23.6\end{array}$ & $\begin{array}{c}\text { Exp. 1: } \\
6.407 \\
\text { Exp. 2: } \\
6.135\end{array}$ & \begin{tabular}{|c|} 
Exp. 1: \\
$11.2-16.2$ \\
Exp. 2: \\
$9.3-10.1$ \\
Exp. 3: \\
$13.8-16.6$ \\
\end{tabular} & \begin{tabular}{|l|}
$9.4-23.1$ \\
\end{tabular} & \begin{tabular}{|c|} 
Exp. 1: \\
$11.7-17.7$ \\
Exp. 2: \\
$14.8-18.4$ \\
Exp. 3: \\
$7.1-11.6$ \\
\end{tabular} & $8.9-27$ & \begin{tabular}{|c} 
Exp. 1: \\
20.2-21.6 \\
Exp. 2: \\
19.3-22.9 \\
Exp. 3: \\
13.3-13.9
\end{tabular} & \begin{tabular}{|c|} 
Exp. 1: \\
$11.6-16.9$ \\
Exp. 2: \\
$12.2-17.3$
\end{tabular} \\
\hline $\begin{array}{c}\text { Maximum } \\
\text { values of } \\
\text { heat transfer } \\
\text { coefficient } \\
\alpha\left[\mathrm{kW} /\left(\mathrm{m}^{2} \mathrm{~K}\right)\right]\end{array}$ & $1.05-1.33$ & $\begin{array}{l}0.36, \\
0.53\end{array}$ & $\begin{array}{c}\text { Exp. 1: } \\
0.2 \\
\text { Exp. 2: } \\
0.325\end{array}$ & \begin{tabular}{|c|} 
Exp. 1: \\
$0.32-0.5$ \\
Exp. 2: \\
$0.202-0.22$ \\
Exp. 3: \\
$0.375-500$ \\
\end{tabular} & $0.19-0.56$ & \begin{tabular}{|c|} 
Exp. 1: \\
$0.32-0.55$ \\
Exp. 2: \\
$0.4-0.5$ \\
Exp. 3: \\
$0.2-0.27$ \\
\end{tabular} & $10-80$ & \begin{tabular}{|c|} 
Exp. 1: \\
100-175 \\
Exp. 2: \\
$70-130$ \\
Exp. 3: \\
20-33 \\
\end{tabular} & \begin{tabular}{|c|} 
Exp. 1: \\
$50-70$ \\
Exp. 2: \\
$60-65$
\end{tabular} \\
\hline $\begin{array}{c}\text { Pressure at } \\
\text { minichannel } \\
\text { inlet } \\
p_{\text {in }}[\mathrm{kPa}]\end{array}$ & 330 & 190 & $\overline{-}$ & $\begin{array}{l}\text { Exp. 1: } \\
\text { 136 } \\
\text { Exp. 2: } \\
115 \\
\text { Exp. 3: } \\
\text { 120-123 }\end{array}$ & 130 & $\begin{array}{c}\text { Exp. 1: } \\
125 \\
\text { Exp. 2: } \\
140 \\
\text { Exp. 3: } \\
120 \\
\end{array}$ & 125 & \begin{tabular}{|c} 
Exp. 1: \\
125 \\
Exp. 2: \\
145 \\
Exp. 3: \\
139 \\
\end{tabular} & \begin{tabular}{c|} 
Exp. 1: \\
140 \\
Exp. 2: \\
140
\end{tabular} \\
\hline $\begin{array}{c}\text { Average } \\
\text { mass flux } \\
G\left[\mathrm{~kg} /\left(\mathrm{m}^{2} \mathrm{~s}\right)\right]\end{array}$ & 219 & 412 & $\begin{array}{l}\text { Exp. 1: } \\
\quad 60 \\
\text { Exp. 2: } \\
\quad 44\end{array}$ & $\begin{array}{l}\text { Exp. 1: } \\
\text { 160 } \\
\text { Exp. 2: } \\
\text { 165 } \\
\text { Exp. 3: } \\
\quad 163\end{array}$ & 236 & $\begin{array}{c}\text { Exp. 1: } \\
211 \\
\text { Exp. 2: } \\
207 \\
\text { Exp. 3: } \\
211\end{array}$ & 285 & $\begin{array}{c}\text { Exp. 1: } \\
204 \\
\text { Exp. 2: } \\
204 \\
\text { Exp. 3: } \\
208\end{array}$ & \begin{tabular}{c|} 
Exp. 1: \\
260 \\
Exp. 2: \\
144
\end{tabular} \\
\hline $\begin{array}{l}\text { Reynolds } \\
\text { number } \\
\text { Re }\end{array}$ & 946 & - & $\begin{array}{l}\text { Exp. 1: } \\
205 \\
\text { Exp. 2: } \\
152\end{array}$ & $\begin{array}{l}\text { Exp. 1: } \\
552 \\
\text { Exp. 2: } \\
\quad 478 \\
\text { Exp. 3: } \\
510\end{array}$ & 735 & $\begin{array}{c}\text { Exp. 1: } \\
704 \\
\text { Exp. 2: } \\
720 \\
\text { Exp. 3: } \\
670 \\
\end{array}$ & 880 & $\begin{array}{c}\text { Exp. 1: } \\
755 \\
\text { Exp. 2: } \\
758 \\
\text { Exp. 3: } \\
714\end{array}$ & \begin{tabular}{c|} 
Exp. 1: \\
1003 \\
Exp. 2: \\
968
\end{tabular} \\
\hline $\begin{array}{c}\text { Inlet liquid } \\
\text { subcooling } \\
\Delta T_{\text {sub,in }}[\mathrm{K}]\end{array}$ & 68 & 36 & $\begin{array}{l}\text { Exp. 1: } \\
56.2 \\
\text { Exp. 2: } \\
45\end{array}$ & $\begin{array}{l}\text { Exp. 1: } \\
54 \\
\text { Exp. 2: } \\
55 \\
\text { Exp. 3: } \\
55\end{array}$ & 50 & $\begin{array}{c}\text { Exp. 1: } \\
42 \\
\text { Exp. 2: } \\
43 \\
\text { Exp. 3: } \\
30 \\
\end{array}$ & 42 & $\begin{array}{c}\text { Exp. 1: } \\
44 \\
\text { Exp. 2: } \\
43 \\
\text { Exp. 3: } \\
42\end{array}$ & \begin{tabular}{|l|} 
Exp. 1: \\
38.5 \\
Exp. 2: \\
42.5
\end{tabular} \\
\hline
\end{tabular}




\section{Comparison of the results obtained by Beck's method coupled with the FEMT and those obtained using the one-dimensional method}

The one-dimensional method described by Piasecka et al. (2017) has been employed to verify the results. This method assumes that the whole heat flux supplied to the heated foil $q_{V}$ is transferred to the fluid flowing in the minichannel. The temperature measured at the surface $y=\delta_{G}$ is assumed to be the temperature of the wall $y=\delta_{G}+\delta_{F}$. This approach is appropriate only when the foil thickness $\delta_{F}$ is negligible. In the one-dimensional method, the heat transfer coefficients have been calculated from the formula (Piasecka et al., 2017)

$$
\alpha_{1 D}^{i}\left(x_{p}\right)=\frac{I \Delta U}{A_{F}\left[T_{p}-T_{f}\left(x_{p}\right)\right]} \quad p=1,2, \ldots, P \quad i=\text { Raw, Smoo }
$$

where $I, \Delta U, A_{F}, T_{f}, T_{p}, i$ have the same denotations as in expressions Eqs. (2.1), (3.1), (3.4), (5.1).

The calculations have been performed using the raw and smoothed measurement data.

The relative differences between the values of the heat transfer coefficient determined with the one-dimensional method and those obtained by means of Beck's method coupled with the FEMT have been calculated from the following formula

$$
\sigma_{i}^{L 1=j}=\frac{1}{P} \sum_{p=1}^{P} \sqrt{\frac{\left[\alpha_{1 D}^{i}\left(x_{p}\right)-\alpha_{2 D}^{i, L 1=j}\left(x_{p}\right)\right]^{2}}{\left[\alpha_{2 D}^{i, L 1=j}\left(x_{p}\right)\right]^{2}}} \quad \text { for } \quad j=10,20 ; \quad i=\text { Raw, Smoo }
$$

where $P, \alpha_{2 D}^{i, L 1=10}, \alpha_{2 D}^{i, L 1=20}$, and $i$ have the same denotations as in formula (5.1), $\alpha_{1 D}^{R a w}$ and $\alpha_{1 D}^{S m o o}$ are values of the heat transfer coefficient obtained by the one-dimensional method using the raw measurement data and the smoothed measurement data, respectively, Eq. (6.1).

The calculation results are presented in Table 4 . The greatest differences between the values of the heat transfer coefficient obtained with the one-dimensional method and those reported for Beck's method coupled with the FEMT occurred at setting \#11 when the raw measurement data was used and the domain was partitioned into $L 1=10$ subdomains in the $x$-direction, see Fig. 6.

Table 4. Relative differences between the values of the heat transfer coefficient obtained with

\begin{tabular}{|c|c|c|c|c|c|c|c|c|c|c|c|c|}
\hline & \multicolumn{6}{|c|}{ Subcooled boiling region } & \multicolumn{6}{|c|}{ Saturated nucleate boiling region } \\
\hline & \multicolumn{12}{|c|}{ Setting number } \\
\hline & $\# 1$ & $\# 2$ & $\# 3$ & $\# 4$ & $\# 5$ & $\# 6$ & $\# 7$ & $\# 8$ & $\# 9$ & \#10 & $\# 11$ & $\# 12$ \\
\hline$\sigma_{\text {Raw }}^{L 1=10}[\%]$ & 1.72 & 1.9 & 1.85 & 1.91 & 1.5 & 1.4 & 12.1 & 9.18 & 11.34 & 10.56 & 13.24 & 12.33 \\
\hline$\sigma_{\text {Raw }}^{L 1=20}[\%]$ & 1.35 & 1.66 & 1.57 & 2.6 & 2.24 & 1.49 & 10.56 & 8.43 & 8.79 & 9.14 & 8.23 & 11.96 \\
\hline$\sigma_{\text {Smoo }}^{L 1=10}[\%]$ & 0.3 & 0.33 & 0.38 & 0.49 & 0.6 & 0.32 & 6.8 & 4.87 & 5.67 & 4.87 & 4.0 & 2.96 \\
\hline$\sigma_{\text {Smoo }}^{L 1=20}[\%]$ & 0.31 & 0.35 & 0.39 & 0.49 & 0.59 & 0.31 & 6.48 & 5.54 & 5.48 & 4.48 & 4.01 & 2.99 \\
\hline
\end{tabular}
the one-dimensional method and those reported for Beck's method coupled with the FEMT

\section{Conclusions}

This paper discusses the application of Beck's method combined with the FEMT to calculate the local values of the heat transfer coefficients for the heat transfer between the heated foil and the fluid flowing in the minichannel. The sensitivity coefficients are introduced as derivatives 


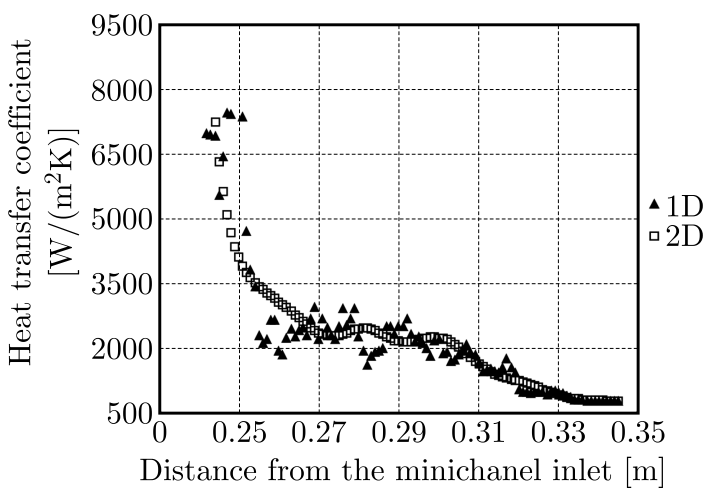

Fig. 6. Heat transfer coefficients at setting \#11 vs. distance from the minichannel inlet obtained by means of the one-dimensional method and Beck's method coupled with the FEMT using the raw measurement data for the domain partitioned into $L 1=10$ subdomains in the $x$-direction

with respect to the unknown heat flux at the edge in order to directly calculate the values of the heat flux with no need to differentiate the temperature functions.

The calculations have been performed using both the raw and smoothed measurement data. In both cases, the boundary $y=\delta_{G}+\delta_{F}, x \in\left\langle x_{1}, x_{P}\right\rangle$ was partitioned into $L 1=10$ and $L 1=20$ subdomains.

Partitioning of the domain in the $x$-direction does not cause considerable changes in the values of the heat transfer coefficient calculated in the subcooled boiling region (the maximum relative difference is approximately $3 \%$, see Table 2 and Fig. 3). However, changes in the values of this coefficient are reported in the saturated nucleate boiling region. Further partitioning in the $x$-direction has a significant influence on the values of this coefficient when raw data rather than smoothed is used, see Table 2 and Fig. 4.

The local values of the heat transfer coefficients are relatively low in the subcooled boiling region (like in Grysa et al., 2012; Hożejowska et al., 2009; Ozer et al., 2011; Piasecka and Maciejewska, 2012, 2013, 2015; Piasecka and Maciejewska, 2015) and high in the saturated nucleate boiling region (like in Hożejowska and Piasecka, 2014; Piasecka and Maciejewska, 2015; Piasecka et al., 2017), see Figs. 3-5.

The values and distribution of the coefficient obtained by means of the proposed method are similar to those reported for a simple, one-dimensional method, see Fig. 6. The relative differences between the coefficients obtained with the two methods, given in Table 4, does not exceed $2.6 \%$ in the subcooled boiling region. In the saturated nucleate boiling region, however, they are greater and reach approximately 13.5\%. Further partitioning of the domain, i.e. from $L 1=10$ into $L 1=20$ subdomains, contributes to reduction in the differences in the values of the coefficients obtained with both approaches only in the saturated nucleatesboiling region when the raw measurement data is used. The differences are negligible in the subcooled boiling region as well as when the smoothed temperature data is used.

\section{Acknowledgements}

The research reported herein was supported by a grant from the National Scientific Center (No. DEC-2013/09/B/ST8/02825).

\section{References}

1. Beck J.V., Blackwell B., Clair C.R.St., 1985, Inverse Heat Conduction. Ill-Posed Problems, Wiley-Interscience Publ., New York 
2. BECK J.V., 1970, Nonlinear estimation applied to the nonlinear inverse heat conduction problem, International Journal of Heat and Mass Transfer, 13, 703-716

3. CiaŁkowski M.J., 2002, New type of basic functions of FEM in application to solution of inverse heat conduction problem, Journal of Thermal Science, 11, 163-171

4. Ciąkowski M.J., Grysa K., 2010, Trefftz method in solving the inverse problems, Journal Inverse Ill-Posed Problems, 18, 595-616

5. Ciąkowski M.J., Frąckowiak A., 2002, Solution of the stationary 2D inverse heat conduction problem by Treffetz method, Journal of Thermal Science, 11, 148-162

6. Duda P., TAler J., 2009, A new method for identification of thermal boundary conditions in water-wall tubes of boiler furnaces, International Journal of Heat and Mass Transfer, 52, $1517-1524$

7. Grysa K., Hożejowska S., Maciejewska B., 2012, Compensatory calculus and Trefftz functions applied to local heat transfer coefficient determination in a minichannel, Journal of Theoretical and Applied Mechanics, 50, 087-1096

8. Grysa K., Maciejewska B., 2013, Trefftz functions for the non-stationary problems, Journal of Theoretical and Applied Mechanics, 51, 251-264

9. Herrera I., 2000, Trefftz method: a general theory, Numerical Methods for Partial Differential Equations, 16, 561-580

10. Hożejowska S., Maciejewska B., Poniewski M.E., 2015, Numerical analysis of boiling twophase flow in mini- and microchannels, [In:] Encyclopedia of Two-Phase Heat Transfer and Flow. I. Fundamentals and Method, Thome J.R. (Edit.), World Scientific Publishing Co Ltd., New Jersey

11. Hożejowska S., Piasecka M., 2014, Equalizing calculus in Trefftz method for solving twodimensional temperature field of FC-72 flowing along the minichannel, Heat and Mass Transfer, 50, 1053-1063

12. Hożejowska S., Piasecka M., Poniewski M.E., 2009, Boiling heat transfer in vertical minichannels. Liquid crystal experiments and numerical investigations, International Journal of Thermal Sciences, 48, 1049-1059

13. Kincaid D., Cheney W., 2002, Numerical Analysis: Mathematics of Scientific Computing, 3rd ed., Brooks/Cole Publishing Company, Belmont, California

14. Kompis V., Konkol F., VAsko M., 2001, Trefftz-polynomial reciprocity based FE formulations, Computer Assisted Mechanics and Engineering Sciences, 8, 385-395

15. Kruk B., SokaŁa M., 1999, Sensitivity coefficients and heat polynomials in the inverse heat conduction problems, Journal of Applied Mathematics and Mechanics, ZAMM, 3, 693-694

16. Kruk B., SokaŁa M., 2000, Sensitivity coefficients applied to two-dimensional transient inverse heat conduction problems, Journal of Applied Mathematics and Mechanics, ZAMM, 81, 945-946

17. KurPisz K., NowAK A.J., 1992, BEM approach to inverse heat conduction problem, Engineering Analysis with Boundary Elements, 10, 291-297

18. Le Niliot C., Lefevre F., 2004, A parameter estimation approach to solve the inverse problem of point heat sources identification, International Journal of Heat and Mass Transfer, 47, 827-841

19. Li Z.-C., Lu T.-T., Huang H.-T., Cheng A.H.-D., 2006, Trefftz, collocation, and other boundary methods - a comparison, Numerical Methods for Partial Differential Equations, 23, 1-52

20. Lin D.T.W., YAN W.-M., Li H.-Y., 2008, Inverse problem of unsteady conjugated forced convection in parallel plate channels, International Journal of Heat and Mass Transfer, 51, 993-1002

21. Maciejewska B., 2004, Application of the modified method of finite elements for identification of temperature of a body heated with a moving heat source, Journal of Theoretical and Applied Mechanics, 42, 771-787 
22. MACIAqG A., 2011, The usage of wave polynomials in solving direct and inverse problems for two-dimensional wave equation, International Journal for Numerical Methods in Biomedical Engineering, 27, 1107-1125

23. Ozer B.A., Oncel A.F., Hollingsworth D.H., Witte L.C., 2011, A method of concurrent thermographic-photographic visualization of flow boiling in a minichannel, Experimental Thermal and Fluid Science, 35, 1522-1529

24. PiaseckA M., 2013, Determination of the temperature field using liquid crystal thermography and analysis of two-phase flow structures in research on boiling heat transfer in a minichannel, Metrology and Measurement Systems, XX, 205-216

25. PiaseckA M., 2014a, Flow boiling heat transfer in a minichannel with enhanced heating surface, Heat Transfer Engineering, 35, 903-912

26. PiaseckA M., 2014b, Laser texturing, spark erosion and sanding of the surfaces and their practical applications in heat exchange devices, Advanced Material Research, 874, 95-100

27. PiaseckA M., 2014c, The use of enhanced surface in flow boiling heat transfer in a rectangular minichannels, Experimental Heat Transfer, 27, 231-255

28. PiAsECKA M., 2015, Impact of selected parameters on boiling heat transfer and pressure drop in minichannels, International Journal of Refrigeration, 56, 198-212

29. Piasecka M., Maciejewska B., 2012, The study of boiling heat transfer in vertically and horizontally oriented rectangular minichannels and the solution to the inverse heat transfer problem with the use of the Beck method and Trefftz functions, Experimental Thermal and Fluid Science, 38, 19-32

30. Piasecka M., Maciejewska B., 2013, Enhanced heating surface application in a minichannel flow and the use of the FEM and Trefftz functions for the solution of inverse heat transfer problem, Experimental Thermal and Fluid Science, 44, 23-33

31. Piasecka M., Maciejewska B., 2015, Heat transfer coefficient during flow boiling in a minichannel at variable spatial orientation, Experimental Thermal and Fluid Science, 68, 459-467

32. Piasecka M., StrąK K., Maciejewska B., 2016, Calculations of flow boiling heat transfer in a minichannel using data from LCT and IRT, Heat Transfer Engineering, 38, 3, 332-346, http://dx.doi.org/10.1080/01457632.2016.1189272

33. Shi J., WANG J., 2009, Inverse problem of transpiration cooling for estimating wall heat flux by LTNE model and CGM method, International Journal of Heat and Mass Transfer, 52, 2714-2720

34. Tikhonov A.N., Arsenin V.Y., 1977, Solution of Ill-Posed Problems, Wiley, New York

35. Trefftz E., 1926, Ein Gegenstück zum Ritzschen Verfahren, 2 Int. Kongress für Technische Mechanik, Zürich, 1926, 131-137

36. 36 Tseng A.A., Chen T.C., Zhao F.Z., 1995, Direct sensitivity coefficient method for solving two-dimensional inverse heat conduction problems by finite-element scheme, Numerical Heat Transfer, Part B: Fundamentals, 27, 291-307

37. Tseng A.A., Chen T.C., Zhao F.Z., 1996, Multidimensional inverse transient heat conduction problems by direct sensitivity coefficent method using a finite-element scheme, Numerical Heat Transfer, Part B: Fundamentals, 29, 365-38 